\title{
Null subspace-based damage detection of structures using vibration measurements
}

\author{
Ai-Min Yan*, Jean-Claude Golinval \\ Department of Aerospace, Mechanics and Materials, Vibration and Identification of Structures, University of Liege, \\ Chemin des Chevreuils 1, B52, B-4000 Liege, Belgium
}

Received 8 June 2004; received in revised form 21 April 2005; accepted 22 April 2005

Available online 24 June 2005

\begin{abstract}
A damage detection method of mechanical system based on subspace identification concepts and statistical process techniques is presented. The aim is to propose a method that is sensitive to small-sized structural damages and suitable for on-line monitoring. Measured time-responses of structures subjected to artificial or environmental vibrations are assembled to form the Hankel matrix, which is further factorised by performing singular-value decomposition to obtain characteristic subspaces. It may be demonstrated that the structural responses are mainly located in the active subspace defined by the first principal components, which is orthonormal to the null subspace defined by the remaining principal components. If no structural damage occurs, the orthonormality relation between the subspaces remains valid with small residues when consecutive data sets are compared, and these residues may be evaluated by the proposed damage indicators. The method is validated using an experimental mock-up of an airplane subjected to different levels of damages simulated. It is also applied in environmental vibration testing of a street lighting device to monitor structural fatigue evolution.
\end{abstract}

(C) 2005 Elsevier Ltd. All rights reserved.

Keywords: Structural health monitoring; Damage detection; Stochastic subspace identification; Null subspace analysis; Fatigue vibration testing

\footnotetext{
${ }^{*}$ Corresponding author. Tel.: +3243669513 ; fax: +3243664856 .

E-mail address: am.yan@ulg.ac.be (Ai-Min Yan).
} 


\section{Introduction}

Early diagnosis of structural damages or machinery malfunctions allows to reduce the maintenance cost of systems and to increase their reliability and safety. Visual and systematic inspections may be periodically performed, sometimes requiring shutdown and disassembly of the system. In many practical situations, it is of interest to use condition-based inspections by continuous or periodical vibration measurements. The combined application of these two kinds of inspections allows to make an optimal maintenance decision. In developing an on-line monitoring tool, both efficiency and simplicity are pursued.

The aim of this paper is to address the damage detection problem by statistical analysis on output-only measurements of structures. The method requires neither an identification of modal parameters nor a construction of a finite element model. Several techniques exist to satisfy partially or completely this objective. For instance, Basseville et al. [1] proposed a subspace identification-based fault detection algorithm using a $\chi^{2}$-type test; Worden et al. [2] developed a novelty analysis for damage detection, which was also used by Sohn and Farrar [3] with a twostage (AR-ARX) prediction model; principal component analysis (PCA) was applied by Friswell and Inman [4] for the sensor validation; this technique has also been used in [5,6] for detection and localisation of structural damage, and the method has been further enhanced in [7] to improve its efficiency. A novelty analysis based on the Kalman model was recently proposed in [8]. The basic assumption is that there is a significant increase in the residual errors when a Kalman model identified from the undamaged system is used to predict responses of the damaged system. Besides, special effort has been paid to eliminate the effect of varying environmental conditions (e.g. temperature variations) on damage detection during a long-term monitoring $[9,10]$.

The present work provides an alternative method to the above developments. It concerns mainly the damage detection problem, which is basically important in some practical situations. Further, damage localisation may be realised by assessing structural matrix information when using enough measuring sensors, see $[11,12]$ as example. The severity of damage may also be approximately assessed by relating the damage indicators with simulated damage cases. The key idea of the proposed method relies on the concepts of subspace identification and null subspace. The response data (such as displacements, velocities, accelerations, stresses, etc.) periodically collected from the monitored structure are used to construct the Hankel matrices. If no structural damage occurs, the orthonormality assumption between the subspaces of the Hankel matrices corresponding to different data sets remains approximately valid according to small residues, which in turn may serve as damage-sensitive features. The threshold level of the residues may be determined by monitoring the healthy structure in various excitation conditions. Several damage indicators are proposed to assess the residues for the damage detection. This null-subspace-based damage detection method is expected to be sensitive to small and local damages of structures and suitable for on-line monitoring in controlled environmental conditions. The efficiency of the proposed method will be illustrated on several experimental applications.

\section{Null subspace analysis of Hankel matrix}

The proposed method is partly inspired by the concept of subspace identification [13] but it does not require modal identification. Let us consider the discrete-time state-space model of a structure 
in the form

$$
\begin{aligned}
& \mathbf{x}_{k+1}=\mathbf{A} \mathbf{x}_{k}+\mathbf{w}_{k}, \\
& \mathbf{y}_{k}=\mathbf{C} \mathbf{x}_{k}+\mathbf{v}_{k},
\end{aligned}
$$

where $\mathbf{x}_{k} \in \Re^{n}$ is the state vector at time step $k$ ( $n$ is the system order), $\mathbf{y}_{k} \in \Re^{m}$ the output vector ( $m$ is the number of output measuring sensors). Matrices $\mathbf{A} \in \Re^{n \times n}$ and $\mathbf{C} \in \mathfrak{R}^{m \times n}$ are called, respectively, the state and output matrices. Vectors $\mathbf{w}_{k}$ and $\mathbf{v}_{k}$ denote, respectively, the state noise and measurement noise processes, which are assumed to be Gaussian white-noise sequences with zero mean.

The concept of subspace identification for linear systems, which was applied to modal analysis of structures in [13-16], is based on the definition of the Hankel matrix that may be calculated in two ways corresponding to either the covariance-driven or data-driven subspace identification algorithms.

- Covariance-driven Hankel matrix

$$
\mathbf{H}_{p, q}=\left[\begin{array}{ccccc}
\Lambda_{0} & \Lambda_{1} & \ldots & \ldots & \Lambda_{q-1} \\
\Lambda_{1} & \Lambda_{2} & \ldots & \ldots & \Lambda_{q+1} \\
\ldots & \ldots & \ldots & \ldots & \ldots \\
\Lambda_{p-1} & \Lambda_{p} & \ldots & \ldots & \Lambda_{p+q-2}
\end{array}\right] ; q \geqslant p,
$$

where $p, q$ are user-defined parameters (taking $p=q$ in this paper) and $\boldsymbol{\Lambda}_{i}$ represents the output covariance matrix, which may be estimated from a set of $N$ output data samples $\mathbf{y}_{k}$ as

$$
\Lambda_{i} \approx \frac{1}{N-i} \sum_{k=1}^{N-i} \mathbf{y}_{k+i} \mathbf{y}_{k}^{\mathrm{T}} .
$$

This Hankel matrix may be factorised into two subspaces, from which, specially from the left kernel subspace, the modal information may be extracted.

- Data-driven Hankel matrix

$$
\mathbf{H}_{1,2 i}=\left[\begin{array}{cccc}
\mathbf{y}_{1} & \mathbf{y}_{2} & \ldots & \mathbf{y}_{j} \\
\ldots & \ldots & \ldots & \ldots \\
\mathbf{y}_{i} & \mathbf{y}_{i+1} & \ldots & \mathbf{y}_{i+j-1} \\
\hdashline \mathbf{y}_{i+1} & \mathbf{y}_{i+2} & \ldots & \mathbf{y}_{i+j} \\
\ldots & \ldots & \ldots & \ldots \\
\mathbf{y}_{2 i} & \mathbf{y}_{2 i+1} & \ldots & \mathbf{y}_{2 i+j-1}
\end{array}\right] \equiv\left(\begin{array}{c}
\mathbf{Y}_{p} \\
\hdashline \mathbf{Y}_{f}
\end{array}\right) \equiv \text { "past" }
$$

where $2 i$ is the user-defined number of row blocks, and $j$ the number of columns (in practice, $j=N-2 i+1$. The Hankel matrix $\mathbf{H}_{1,2 i} \in \mathfrak{R}^{2 m i \times j}$ is split into a "past" and a "future" part of $i$ block rows. The principal idea in modal analysis is to retain all the information in the past for predicting the future through an orthogonal projection of the row space of "future" outputs into the row space of "past" outputs. 
From the point of view of damage detection, we are not concerned in identifying the modal parameters of the structure. Instead, only relative changes of characteristic features are necessary for structural damage assessment. For this purpose, we propose to use a method based on the null subspace concept of the Hankel matrices [17]. Performing the singular-value decomposition (SVD) on the weighted Hankel matrix (3), we obtain

$$
\overline{\mathbf{H}}=\mathbf{W}_{1} \mathbf{H}_{p, q} \mathbf{W}_{2} \approx\left[\begin{array}{ll}
\mathbf{U}_{1} & \mathbf{U}_{2}
\end{array}\right]\left[\begin{array}{ll}
\mathbf{S}_{1} & 0 \\
0 & 0
\end{array}\right]\left[\begin{array}{ll}
\mathbf{V}_{1} & \mathbf{V}_{2}
\end{array}\right]^{\mathrm{T}}=\mathbf{U}_{1} \mathbf{S}_{1} \mathbf{V}_{1}^{\mathrm{T}},
$$

where $\mathbf{W}_{1}$ and $\mathbf{W}_{2}$ are invertible weighting matrices, as defined in [13] where a discussion about their effects on modal identification was given, in the present work only identity matrices are used for simplicity; diagonal matrix $\mathbf{S}_{1}$ contains $n \approx 2 N_{m}$ ( $N_{m}$ is the number of active modes) non-zero singular values sorted in decreasing order. Note that the SVD technique has been extensively used in both damage detection and localisation, see [1,11] as example for two cases, and it may factorise the data structure into its characterised subspaces.

Eq. (6) allows to write the following relations:

$$
\begin{aligned}
& \mathbf{U}_{1}^{\mathrm{T}} \overline{\mathbf{H}} \mathbf{V}_{1} \approx \mathbf{S}_{1}, \\
& \overline{\mathbf{H}} \mathbf{V}_{2} \approx 0 \text { or } \overline{\mathbf{H}} \mathbf{V}_{2}=\zeta_{v}, \\
& \mathbf{U}_{2}^{\mathrm{T}} \overline{\mathbf{H}} \approx 0 \text { or } \mathbf{U}_{2}^{\mathrm{T}} \overline{\mathbf{H}}=\zeta_{u} .
\end{aligned}
$$

It may be stated that the SVD of the weighted Hankel matrix $\overline{\mathbf{H}} \in \Re^{r \times c}$, where $r=m \times p$ and $c=m \times q$, leads to four fundamental subspaces: $\mathbf{U}_{1}$ contains the maximum number (i.e. $n$ ) of independent column vectors that span the column space of $\overline{\mathbf{H}} ; \mathbf{V}_{1}^{\mathrm{T}}$ contains the maximum number (n) of independent row vectors that span the row space of $\overline{\mathbf{H}} ; \mathbf{U}_{2}$ contains the maximum number $(c-n)$ of independent column vectors that span the column null space of $\overline{\mathbf{H}} ; \mathbf{V}_{2}^{\mathrm{T}}$ contains the maximum number $(r-n)$ of independent row vectors that span the row null space of $\overline{\mathbf{H}}$.

In most cases, the order $n$ of the system is difficult to determine so that Eqs. (7)-(9) merely apply with residual terms. Residue matrices $\zeta$ are mainly due to noise effects and weakly excited high modes that are neglected by cutting off with a chosen value $n$ as assumed system order. It follows that Eqs. (7)-(9) are not ideal candidates for structural damage detection since the amplitude of the residues resulting from erroneous definition of the system order may mask the residues variation due to small structural damages.

On the other hand, due to the orthonormality of matrices $\mathbf{U}$ and $\mathbf{V}$, the following equations are always true for any data set:

$$
\mathbf{U}_{2}^{\mathrm{T}} \mathbf{U}_{1}=0
$$

or

$$
\begin{aligned}
& \mathbf{U}_{2}^{\mathrm{T}}\left(\mathbf{U}_{1} \mathbf{S}_{1} \mathbf{V}_{1}^{\mathrm{T}}\right)=0, \\
& \mathbf{V}_{1}^{\mathrm{T}} \mathbf{V}_{2}=0
\end{aligned}
$$

or

$$
\left(\mathbf{U}_{1} \mathbf{S}_{1} \mathbf{V}_{1}^{\mathrm{T}}\right) \mathbf{V}_{2}=0 .
$$


Comparing Eqs. (11) and (13) with Eqs. (8)-(9), we are using the active part (with the chosen system order $n$ ) of column and row spaces instead of the original Hankel matrix. It is important to note that Eqs. (10)-(13) hold independently of the precise determination of the system order $n$.

In the following, only the left kernel relations (10)-(11) of the Hankel matrix will be considered for damage detection analysis. It may be shown that matrix $\mathbf{U}_{1}$ contains the first $n$ active principal components and constructs a hyperplane around which the response data locate [7]. Without damage or variation of environmental conditions, the active subspace $\mathbf{U}_{1}$ of the Hankel matrix remains unchanged (i.e. no rotation of the hyperplane occurs) and the orthonormality relations (10)-(11) apply approximately between different data sets. Therefore, observing rotation of this subspace or change in orthonormality between different data sets gives damage information of the monitored structure. For convenience of description, $\mathbf{U}_{1}$ and $\mathbf{U}_{2}$ will be called hereafter, respectively, the column active subspace and the column null subspace of the Hankel matrix $\overline{\mathbf{H}}$.

The similar procedure may be applied when the definition (5) of the data-driven Hankel matrix is adopted. As only the left kernel of the Hankel matrix is considered, SVD is performed on the covariance of the Hankel matrix

$$
\overline{\mathbf{H}}_{2 i} \overline{\mathbf{H}}_{2 i}^{\mathrm{T}} \approx\left[\begin{array}{ll}
\overline{\mathbf{U}}_{1} & \overline{\mathbf{U}}_{2}
\end{array}\right]\left[\begin{array}{ll}
\overline{\mathbf{S}}_{1} & 0 \\
0 & 0
\end{array}\right]\left[\begin{array}{ll}
\overline{\mathbf{U}}_{1} & \overline{\mathbf{U}}_{2}
\end{array}\right]^{\mathrm{T}}=\overline{\mathbf{U}}_{1} \overline{\mathbf{S}}_{1} \overline{\mathbf{U}}_{1}^{\mathrm{T}},
$$

where $\overline{\mathbf{H}}_{2 i}=\mathbf{W}_{1} \mathbf{H}_{1,2 i} \mathbf{W}_{2} ; \mathbf{W}_{1}$ and $\mathbf{W}_{2}$ are two weighting matrices (both are chosen as identity matrix in this work), and subscript $2 i$ denotes the row block number. As the dimension of $\overline{\mathbf{H}}_{2 i} \overline{\mathbf{H}}_{2 i}^{\mathrm{T}}$ is much smaller than the dimension of the original matrix $\overline{\mathbf{H}}_{2 i}$, this modification leads to a much lower cost in the SVD calculation. Performing the same analysis as for the covariance-driven Hankel matrix, an expression similar to Eq. (10) is obtained

$$
\overline{\mathbf{U}}_{2}^{\mathrm{T}} \overline{\mathbf{U}}_{1}=0
$$

or

$$
\overline{\mathbf{U}}_{2}^{\mathrm{T}}\left(\overline{\mathbf{U}}_{1} \overline{\mathbf{S}}_{1} \overline{\mathbf{U}}_{1}^{\mathrm{T}}\right)=0 .
$$

Once again, Eqs. (15)-(16) are always true and do not depend on the chosen system order $n$.

\section{Damage detection by null subspace analysis}

\subsection{Residue matrices}

When two different data sets are examined, the first one associated to the column active subspace $\mathbf{U}_{1}$ or active Hankel matrix $\left(\mathbf{U}_{1} \mathbf{S}_{1} \mathbf{U}_{1}^{\mathrm{T}}\right)$, and the second one associated to the column null subspace $\mathbf{U}_{2}$, Eqs. (10)-(11) or (15)-(16) do no longer strictly apply because of noise effects and other error sources (e.g. variation of the excitation). Therefore, a series of tests in structural healthy states should be performed to provide a reference limit of the residues. It is expected that those preliminary tests may be performed with different excitation levels because in most practical situations, the excitations may change from time to time although they may be assumed to remain stationary during short acquisition periods. 
Let us assume that the column null subspace $\mathbf{U}_{2,0}$ has been determined from one reference data set (indicated by subscript 0 ). Now we consider the column active subspace $\mathbf{U}_{1, i}$ (and $\mathbf{S}_{1, i}, \mathbf{V}_{1, i}$ ) corresponding to the $i$ th data set. The residue matrices calculated from Eqs. (10)-(11) or (15)-(16) may be written as

$$
\begin{aligned}
\boldsymbol{\delta}_{i} & =\mathbf{U}_{2,0}^{\mathrm{T}} \mathbf{U}_{1, i}, \\
\Delta_{i} & =\mathbf{U}_{2,0}^{\mathrm{T}}\left(\mathbf{U}_{1, i} \mathbf{S}_{1, i} \mathbf{V}_{1, i}^{\mathrm{T}}\right) .
\end{aligned}
$$

Note that when the data-driven Hankel matrix is used, $\mathbf{V}_{1, i}$ is replaced by $\mathbf{U}_{1, i}$. The residue matrix $\boldsymbol{\delta}_{i}$ represents the orthonormality change between the subspaces of responses due to noise effects and/or mainly due to structural damages if any, while the residue matrix $\Delta_{i}$ represents the orthonormality change weighted by the active singular-value matrix $\mathbf{S}_{1, i}$ and the right kernel matrix $\mathbf{V}_{1, i}^{\mathrm{T}}$. Both residue matrices (17)-(18) may be candidates as damage-sensitive features. From a numerical point of view, however, the former seems better than the latter due to the fact that, although the effect of $\mathbf{V}_{1, r}^{\mathrm{T}}$ is not clear, $\mathbf{S}_{1, r}$ decreases generally with damages and this may reduce the sensitivity of indicator to damage specially when measured data are not well normalised. Nevertheless, it may be shown that when Eq. (18) is adopted for damage detection, the present method may be related in some extent to the method proposed in [1].

\subsection{Damage indicators}

Starting from Eq. (17), which expresses the orthonormality change between two subspaces, we propose to take the complementary angle between subspaces $\mathbf{U}_{2,0}$ and $\mathbf{U}_{1, i}$ as damage indicator

$$
\alpha_{i}=\sin ^{-1}\left[\operatorname{norm}\left(\boldsymbol{\delta}_{i}\right)\right],
$$

where norm (.) is an operator giving the maximal singular value of a matrix. Obviously the value of $\alpha$ remains in the range [0-90 $]$. A large angle value indicates changes or damages of the system. The second possible damage indicator may be given by the norm of matrix $\Delta_{i}$, which expresses the loss of orthonormality in Eq. (18):

$$
\sigma_{i}=\operatorname{norm}\left(\Delta_{i}\right) \text {. }
$$

Another damage indicator $D_{i}$ may be built from the residue matrix by following a procedure used by Basseville et al. [1] and Fritzen et al. [18]. It consists first of transforming the residue matrix into a vector $\{\Delta\}_{i}$ and then into a normalised scalar $D_{i}$ by an inner-product operation of the vector:

$$
D_{i}=\{\Delta\}_{i}^{\mathrm{T}} \Sigma^{-1}\{\Delta\}_{i}
$$

with

$$
\{\Delta\}_{i}=\operatorname{vec}\left(\Delta_{i}\right)
$$

where vec (.) denotes the column stacking operator. The residue covariance matrix $\boldsymbol{\Sigma}$ in Eq. (21) may be estimated using $N_{\text {ref }}$ reference data sets

$$
\boldsymbol{\Sigma}=\frac{1}{N_{\text {ref }}-1} \sum_{i=1}^{N_{\text {ref }}}\{\Delta\}_{i}\{\Delta\}_{i}^{\mathrm{T}} .
$$


However, it should be pointed out the residual covariance matrix $\boldsymbol{\Sigma}$ is singular when the number of reference data sets is lower than the row size of the Hankel matrix (this often happens as the latter is usually large). In order to avoid this difficulty, Eq. (21) may be replaced by

$$
\bar{\Delta}_{i}=\frac{\{\Delta\}_{i}^{\mathrm{T}}\{\Delta\}_{i}}{\Sigma_{\Delta}}
$$

with

$$
\Sigma_{\Delta}=\frac{1}{N_{\text {ref }}} \sum_{j=1}^{N_{\text {ref }}}\{\Delta\}_{j}^{\mathrm{T}}\{\Delta\}_{j},
$$

where $\boldsymbol{\Sigma}_{\Delta}$ becomes a scalar. Similarly, we have

$$
\bar{\delta}_{i}=\frac{\{\delta\}_{i}^{\mathrm{T}}\{\delta\}_{i}}{\Sigma_{\delta}}
$$

with

$$
\begin{aligned}
& \{\delta\}_{i}=\operatorname{vec}\left(\boldsymbol{\delta}_{i}\right), \\
& \Sigma_{\delta}=\frac{1}{N_{\text {ref }}} \sum_{j=1}^{N_{\text {ref }}}\{\delta\}_{i}^{\mathrm{T}}\{\delta\}_{j} .
\end{aligned}
$$

By this modification, $N_{\text {ref }}=1$ may be applied as a simplest case. If the structure remains undamaged, the normalised indicators (24) and (26) should be close to unity. Conversely, it is expected that structural damages will cause obvious increase of the proposed indicators.

\subsection{Comparison with principal component analysis}

As stated in Section 1, PCA has been used in the field of structural health monitoring for sensor validation and for structural damage detection in constant or varying environmental conditions.

PCA of the sensor time-responses allows to extract principal directions defining a subspace that is representative of the dynamics of the monitored structure. Assuming that each data set is collected in the response matrix $\mathbf{Y}=\left\{\ldots \mathbf{y}_{k} \ldots\right\} \in \Re^{m \times n}$, where $k=1 \ldots N$. By performing SVD so that $\mathbf{Y}=\mathbf{U S V}^{\mathrm{T}}$, we may write the responses spanning the defined subspace

$$
\mathbf{y}(t)=\sum_{i=1}^{N_{m}} a_{i}(t) \mathbf{U}_{1}(:, i)+\mathbf{r},
$$

where $a_{i}$ is a time-varying coefficient related to the $i$ th principal direction. The second term $\mathbf{r}$ is a residue vector when only the first $N_{m}$ principal components are used to reconstruct the response history. It is easy to demonstrate that the adopted subspace is the same as the column active subspace defined in Eq. (6) or (14) if the number of row block of the Hankel matrix is taken equal to 1 . Any change in the response of a single sensor directly affects the subspace spanned by the complete sensor response. Geometrically speaking, the principal directions extracted from the test data construct a hyperplane, as illustrated in Fig. 1 in the 2D case. Specially this hyperplane coincides with the hyperplane defined by the mode-shape functions. The rotation between the 


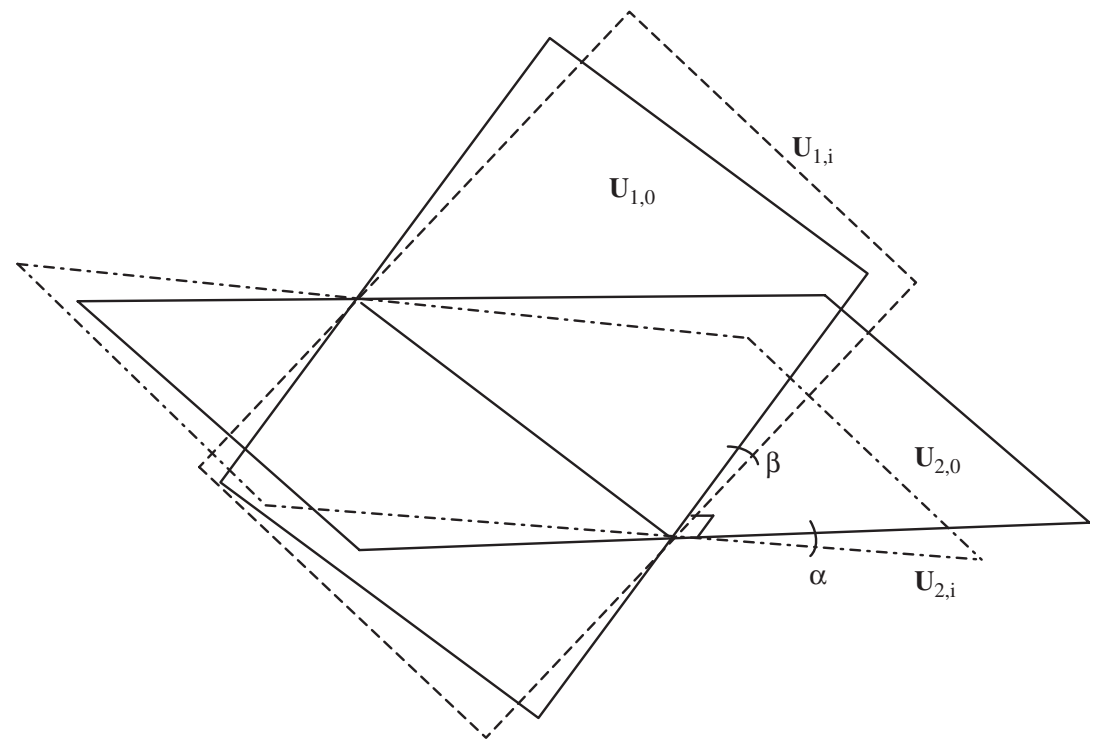

Fig. 1. 2D geometric illustration of damage detection based on PCA or NSA.

active subspaces $\mathbf{U}_{1, i}$ and $\mathbf{U}_{1,0}$ that corresponds, respectively, to the current and initial healthy states of the structure, may be represented by angle $\beta$ between the two subspaces as shown in Fig. 1.

On the other hand, the aim of null subspace analysis (NSA) is to evaluate the change of orthonormality between the column active subspace $\mathbf{U}_{1,0}$ of the reference data and the column null subspace $\mathbf{U}_{2, i}$ of the current data. Using Eq. (19) as damage indicator corresponds to observing the complementary angle $\alpha$ between subspaces $\mathbf{U}_{1,0}$ and $\mathbf{U}_{2, i}$. Due to the orthonormality property of subspaces, the two methods appear to be equivalent if taking only one row block of the Hankel matrix in NSA.

It should be pointed out that the proposed procedure based on the Hankel matrix uses a dynamic modelling in the sense that a time-shift output record is considered in the analysis. It consists of analysing the vibration signal at different instants rather than considering snapshots as in classical PCA. In this manner, the defined hyperplane contains all modal information (e.g. natural frequencies and mode shapes). As a comparison, the hyperplane defined by PCA contains only mode shape information. Additionally, by choose a suitable row block number, NSA can be performed even with only one-sensor-response record using same damage indicators. These improve the application efficiency of the proposed method.

\section{Application examples}

\subsection{Damage detection on an aircraft model}

The tested structure is an aircraft model made of steel and suspended by means of three springs as shown in Fig. 2a. The fuselage consists of a straight beam of rectangular section. Plate-type 


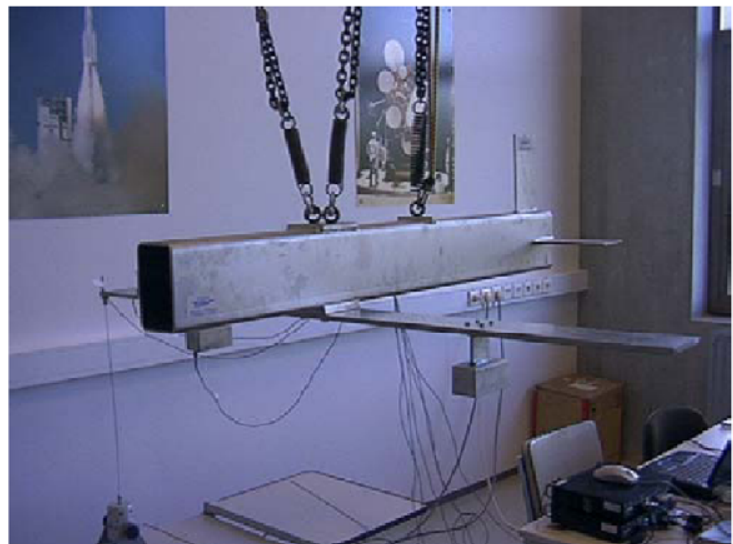

(a)

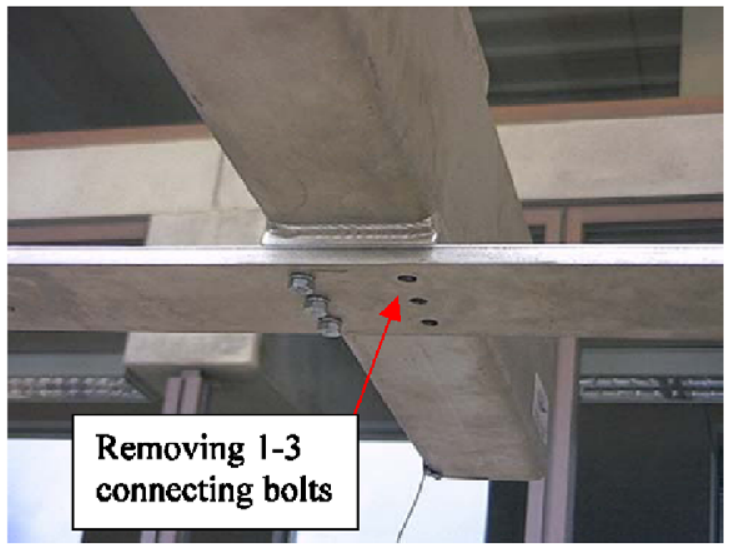

(b)

Fig. 2. (a) Experimental aircraft model and (b) simulated damages: removing 1-3 connecting bolts on the right-hand side of the wing.

beams connected to the fuselage form the wings and tail. The structure is randomly excited on the top left wing by means of an electro-dynamic shaker in the frequency range of $0-130 \mathrm{~Hz}$. The dynamic responses of the model are captured by 11 accelerometers installed on the wings and tail. As a statistical method, the number of data point needed depends on the testing conditions (the excitation stationariness and noise level). In this test, each data set consists of $8192 \times 11$ points. Three levels of damage are created by removing, respectively, one, two and three connecting bolts on the right-hand side of the wing attachment as shown in Fig. 2 b.

The experiment was used previously to illustrate the application of damage detection methods based on PCA and the Kalman model [7,8]. The same data are now used to illustrate improvements of the present method based on NSA in comparison with PCA. First, damage detection is performed for various reference (healthy) states consisting of different levels of excitation; then, it is applied to examine three levels of damages. When using the NSA-based method, the row block number of the Hankle matrix ( $p$ or $2 i$ ) was taken equal to 10. The singular value diagram of Fig. 3 helps us to choose an appropriate order for the active column subspace. Several rules can be applied for selecting the number of principal components (i.e. order $n$ ) to be retained [19]. Here, the order was finally chosen equal to 10 since it corresponds to an accumulated percentage of variance in the range $80-90 \%$ and the next mode (corresponding to order 11) shows an evident decrease of variance energy with respect to mode 10 .

Table 1 and Fig. 4 compare the subspace angles as damage indicator obtained by NSA and PCA, respectively. The detection results are also presented in Fig. 5 where using the normalised indicators (24) denoted by $H-x^{2}$, and Eq. (26) denoted by $x^{2}$. The results obtained with the two kinds of the Hankel matrix, respectively, i.e. data-driven (denoted by "data_") and covariancedriven (denoted by "COV_,"), and with three different indicators are generally consistent. In the reference state, despite of a large difference in excitation levels (for example, in test case 2, the excitation level ratio is $0.5: 1.5)$, the identified subspace angle or other indicators remain relatively small. Conversely, a very slight damage of Level 1 leading to a decrease of about $0.6 \%$ of the first natural frequency, already causes a significative increase of the indicators. The damage indicators 


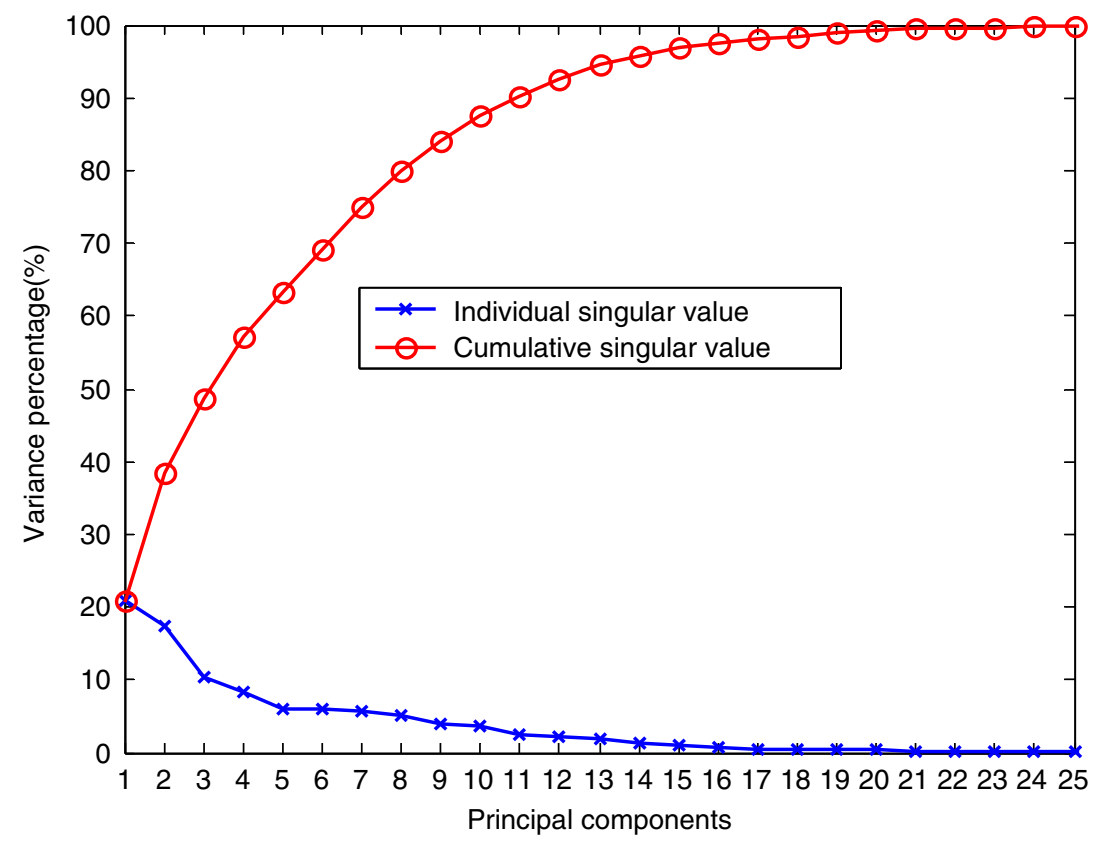

Fig. 3. Singular values of Hankel matrix vs. the number of principal components.

Table 1

Tests on aircraft model taking subspace angle as damage indicator by NSA and PCA

\begin{tabular}{|c|c|c|c|c|c|c|c|}
\hline \multirow[t]{3}{*}{ Method } & \multicolumn{7}{|l|}{ Test case } \\
\hline & 1 & 2 & 3 & 4 & 5 & 6 & 7 \\
\hline & Excit. 1:1 & Excit.0.5:1.5 & Excit.1:1.5 & Excit.1:0.5 & Level 1 & Level 2 & Level 3 \\
\hline $\begin{array}{l}\text { NSA-angle }\left({ }^{\circ}\right) \\
\text { CVA_Hankel }\end{array}$ & 2.91 & 5.76 & 7.62 & 9.20 & 24.82 & 75.95 & 77.99 \\
\hline $\begin{array}{l}\text { NSA-angle }\left({ }^{\circ}\right) \\
\text { Data_Hankel }\end{array}$ & 2.78 & 5.70 & 6.80 & 9.28 & 20.09 & 76.10 & 78.18 \\
\hline $\begin{array}{l}\text { PCA-angle }\left({ }^{\circ}\right) \\
(I=1, n=5)\end{array}$ & 2.895 & 2.892 & 5.78 & 8.143 & 5.74 & 39.95 & 33.37 \\
\hline
\end{tabular}

Note: case 1-4 for reference tests with different excitation ratios; case 5-7 for damaged structure.

increase generally with the damage levels. The results show a general improvement of the NSAbased method to the PCA-based one.

\subsection{Fatigue testing of a street-lighting device}

In order to simulate the effect of wind-induced vibrations on a street-lighting device and to assess its mechanical integrity, fatigue vibration testing is usually performed on electro-dynamic 


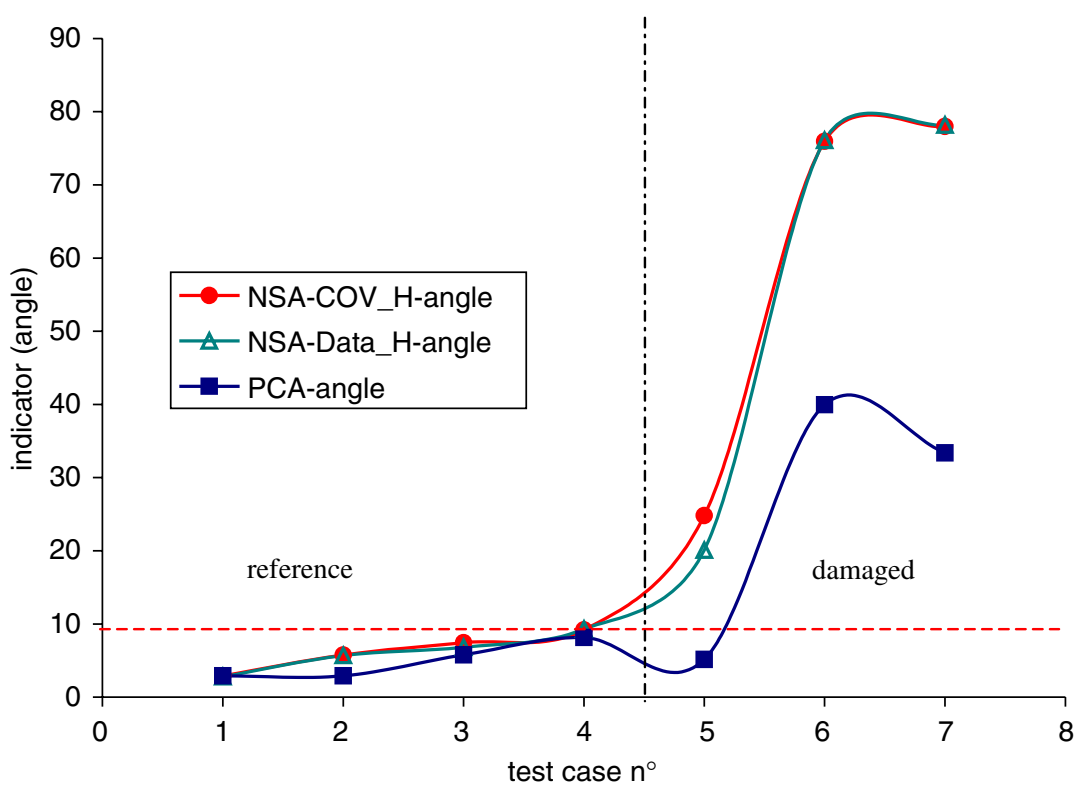

Fig. 4. Subspace angles by NSA and PCA as damage indicator.

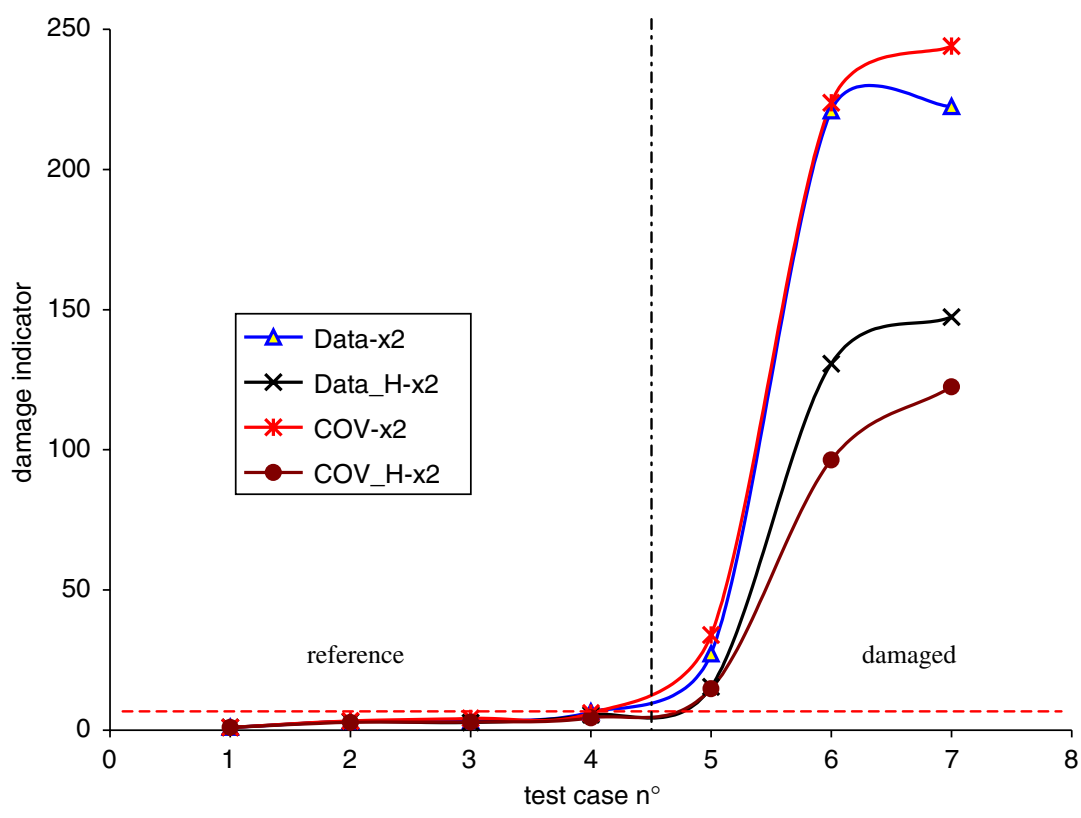

Fig. 5. Normalised damage indicators increase with damage levels.

shaker as illustrated in Fig. 6. The developed NSA method has been applied to monitor the damage evolution during such a vibration fatigue test. According to the Belgian testing standard, a lighting device tested should be vibrated at its first natural frequency during $1 \mathrm{~h}$ with 
acceleration amplitude of $0.5 \mathrm{~g}$. Ten accelerometers were installed at different locations to monitor the structure with a sampling frequency of $256 \mathrm{~Hz}$. In order to be able to easily observe damage, vibration testing was performed during $4 \mathrm{~h}$ instead. It should be noted that the first natural frequency of the lighting device decreases gradually during the test because of the apparition and the growth of damage, and the controlling system of the shaker allows to follow this variation. At the end of the test, cracks appear close to the attachment of the device as illustrated in Fig. 7.

The response records are collected in consecutive windows of $8192 \times 10$ points and the NSA is then performed on each window. The difference between the reference data (taking the first blocks of data) and each current block of observations is checked by evaluating the characteristic angle (19), or the normalised indicators given by Eqs. (24) and (26). For this special application, the results obtained with different number of row block ( $p$ or $2 i$ ) are similar. Simply the monitoring results with one row block are presented, so that the methods based on the COV-driven or datadriven Hankel matrix are equivalent. It may be seen from Fig. 8 that only one principal

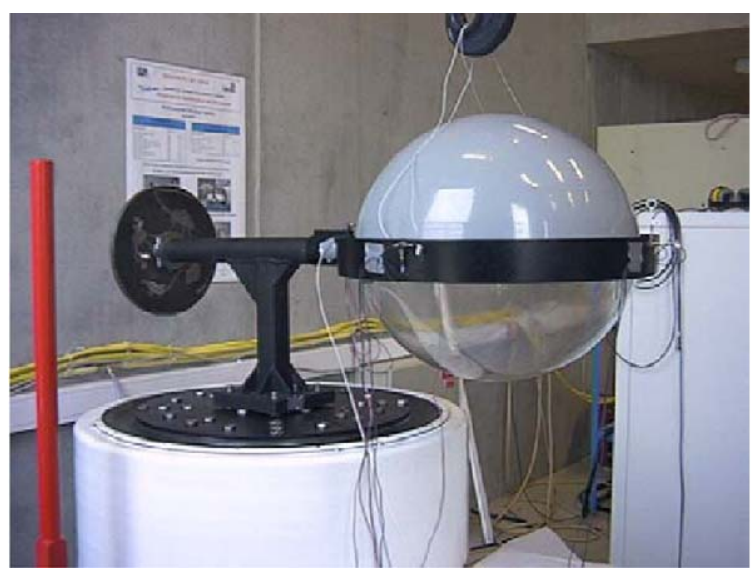

Fig. 6. Fatigue vibration testing of street-lighting device.

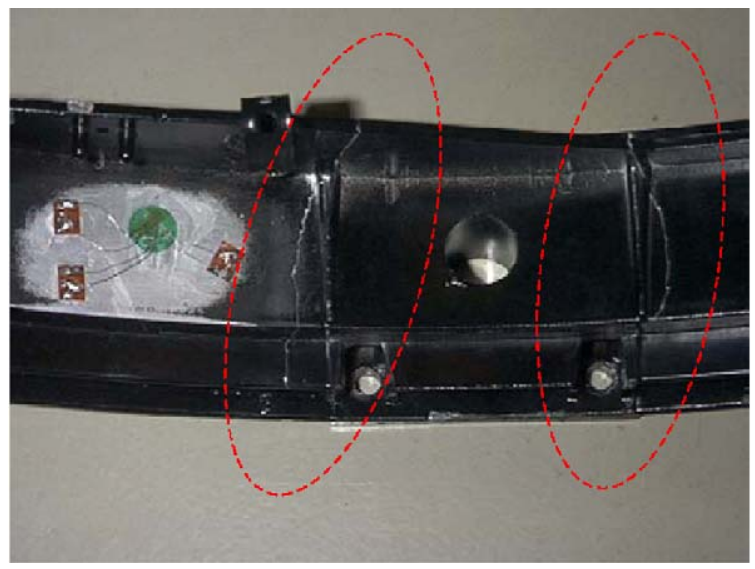

Fig. 7. View of the cracks. 
component is necessary to define the active subspace as it contains about $80 \%$ of the energy. This is reasonable as the structure was excited at the first resonance mode. In the first hours of the test, the damage indicator (i.e. the angle) increases slightly and linearly, but no observable crack appears at this stage. Similarly, the damage indicator continues to increase in a stable way during most of the last hour (Fig. 9). At the last stage of the test (i.e. the last 15 data blocks corresponding to about $8-10 \mathrm{~min}$ ), damage accelerates with the crack growth. Similar results are obtained with the other normalised indicators (Fig. 10).

The time-evolution of the natural frequency of the first mode is also recorded as shown in Fig. 11, and it can be compared with the NSA detection results showing a good agreement.

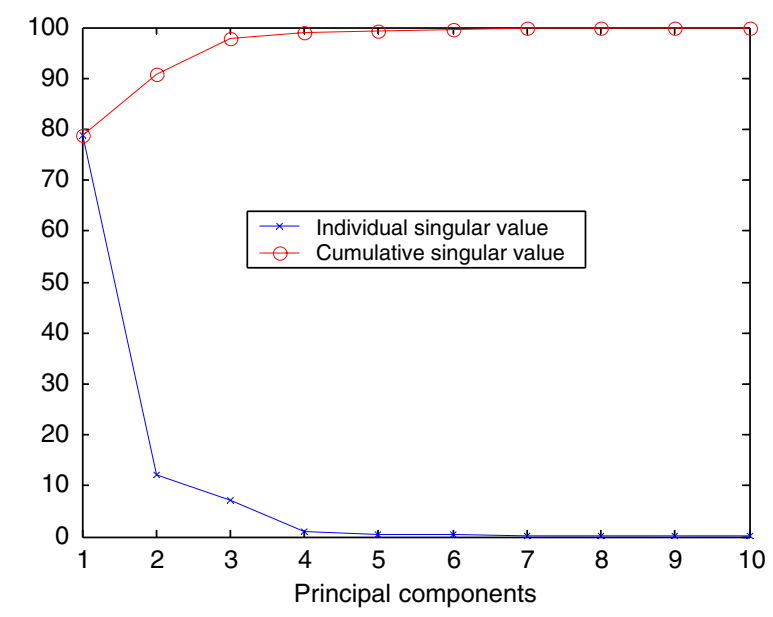

Fig. 8. Singular-value diagram (row block number $p=1$ ).

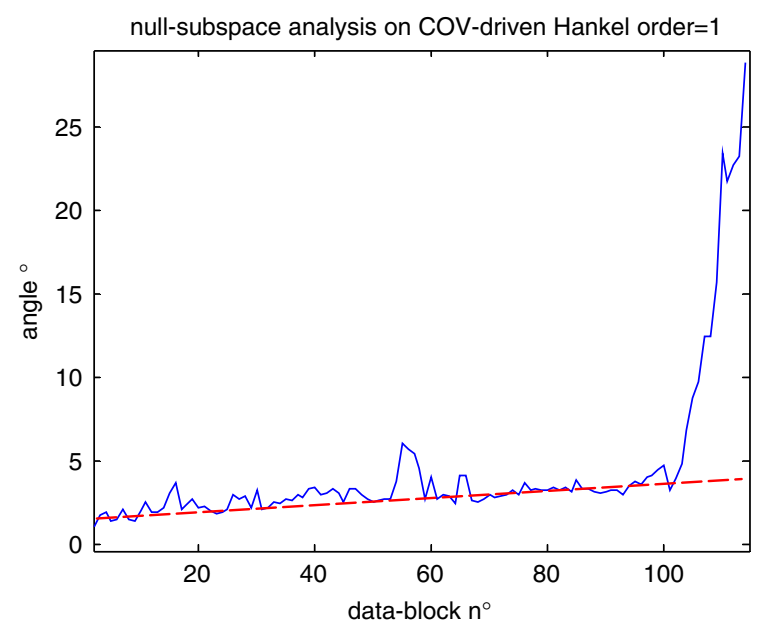

Fig. 9. Damage indicator (angle) in the last hour. 


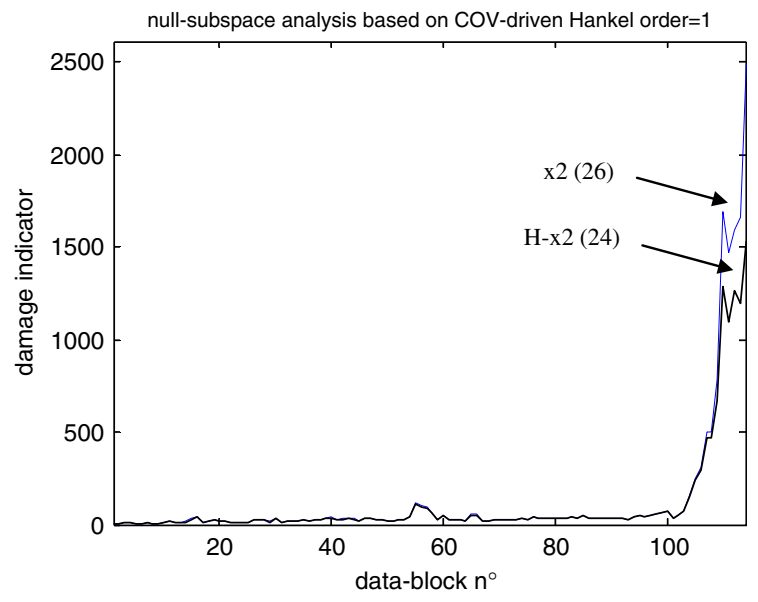

Fig. 10. Normalised indicators in the last hour.

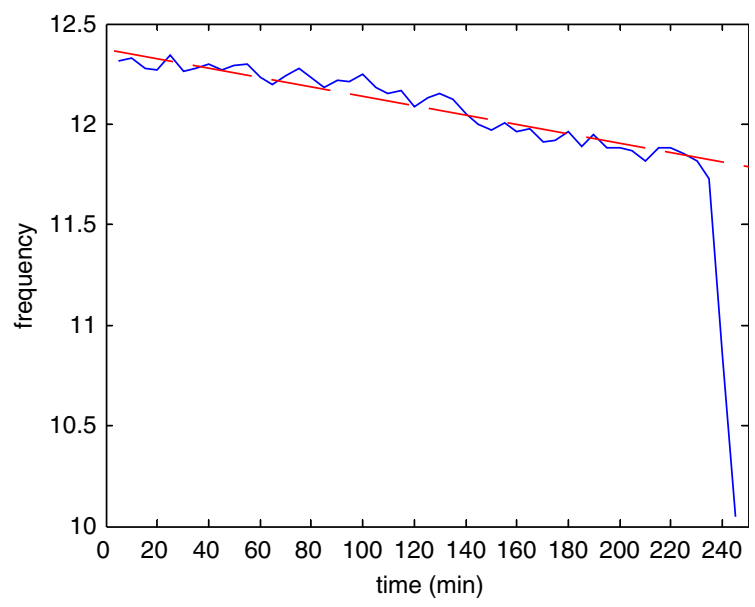

Fig. 11. Time-evolution of the first natural frequency.

\section{Conclusions}

The damage detection method proposed in this paper is based on subspace analysis of the Hankel matrices constructed from output-only vibration measurements. It is demonstrated that the column active subspace of the Hankel matrix defined by the first principal components is orthonormal to the column null subspace defined by the remaining principal components. The residues in the orthonormality relation obtained from different data sets (i.e. between the null subspace of the reference data and the active subspace of the current data) may be used to detect possible damage in the structure. Several damage indicators are proposed to characterise the residue matrices, which are expected to be sensitive to small and local structural damages. 
Experimental applications with two kinds of excitation, one is random excitation and the other one following the first resonance mode of the structure, have been used to illustrate the efficiency of the method. Because of its simplicity, the method is expected to be suitable for on-line monitoring of structures in service or vibration fatigue testing.

\section{Acknowledgements}

This work is supported by the Walloon Region government of Belgium (convention RW-ULg No. 9613419), which is gratefully acknowledged.

\section{References}

[1] M. Basseville, M. Abdelghani, A. Benveniste, Subspace-based fault detection algorithms for vibration monitoring, Automatica 36 (2000) 101-109.

[2] K. Worden, G. Manson, N.R.J. Fieller, Damage detection using outlier analysis, Journal of Sound and Vibration 229 (2000) 647-667.

[3] H. Sohn, C.F. Farrar, Damage diagnosis using time series analysis of vibration signals, Smart Materials \& Structures 10 (2001) 1-6.

[4] M.I. Friswell, D.J. Inman, Sensor validation for SMART structures, IMAC XVIII-18th International Modal Analysis Conference, San Antonio, 2000, pp. 483-489.

[5] P. De Boe, J.C. Golinval, Principal component analysis of piezo-sensor array for damage localization, Structural Health Monitoring 2 (2003) 137-144.

[6] P. De Boe, A.M. Yan, J.C. Golinval, Substructure damage detection by principal component analysis: application to environmental vibration testing, Structural Health Monitoring 2003, Stanford, CA, USA, September 15-17, 2003, pp. 642-649.

[7] A.M. Yan, P. De Boe, J.C. Golinval, Structural integral monitoring by vibration measurements, in: G.C. Sih, S.T. Tu, Z.D. Wang (Eds.), FM2003-Structural Integrity and Materials Aging, Shanghai, 2003, pp. $363-370$.

[8] A.M. Yan, P. De Boe, J.C. Golinval, Structural diagnosis by Kalman model based on stochastic subspace identification, Structural Healthy Monitoring 3 (2004) 103-119.

[9] A.M. Yan, G. Kerschen, P. De Boe, J.C. Golinval, Structural damage diagnosis under changing environmental conditions - Part I: linear analysis and Part II: local PCA for nonlinear analysis, Mechanical Systems \& Signal Processing, 19 (2005), pp. 847-864 and pp. 865-880.

[10] A.M. Yan, J.C. Golinval, B. Peeters, G. De Roeck, A comparative study on damage detection of Z24-Bridge: oneyear monitoring with varying environmental conditions, Structural Health Monitoring 2004, Munich, Germany, July 7-9, 2004, pp. 791-799.

[11] D. Bernal, Load vectors for damage localization, Journal of Engineering Mechanics ASCE 128 (2002) 7-14.

[12] A.M. Yan, J.C. Golinval, Structural damage localization by measured flexibility and stiffness via subspace identification, Engineering Structures, Special Issue on Structural Health Monitoring, Damage Detection and Long-Term Performance, in press.

[13] P. Van Overschee, B. De Moor, Subspace Identification for Linear Systems: Theory ImplementationApplications, Kluwer Academic Publishers, Dordrecht, Netherlands, 1996.

[14] B. Peeters, G. De Roeck, Reference-based stochastic subspace identification for output-only modal analysis, Mechanical Systems and Signal Processing 13 (1999) 855-878.

[15] L. Hermans, H.V.D. Auweraer, Modal testing and analysis of structures under operational conditions: industrial applications, Mechanical Systems and Signal Processing 13 (1999) 193-216.

[16] K. De Cock, B. Peeters, A. Vecchio, H.V.D. Auweraer, B. De Moor, Subspace system identification for mechanical engineering, Proceedings of ISMA 2002, pp. 1333-1352. 
[17] J.N. Juang, M.Q. Phan, Identification and Control of Mechanical Systems, Cambridge University Press, Cambridge, 2001, p. 29.

[18] C.P. Fritzen, G. Mengelkamp, A. Güemes, Elimination of temperature effects on the damage detection within a smart structure concept, Structural Health Monitoring 2003, Stanford, CA, USA, September 15-17, 2003, pp. $1530-1538$.

[19] A.C. Rencher, Multivariable Statistical Influence and Applications, Wiley, New York, NY, USA, 1998. 